\title{
Gene therapy in the era of nanotechnology/a review of current data
}

\section{Introduction}

Gene therapy is emerging as a new class of therapeutics for the treatment of inherited and acquired diseases. However, poor cellular uptake and instability of DNA in the physiological milieu limits its therapeutic potential, hence a vector which can protect and efficiently transport DNA to the target cells must be developed. Nanotechnologybased non-viral vectors have been proposed as potential candidates.

\section{Nanoparticles used in gene delivery}

Polymer nanoparticles (PNPs) deliver genes or therapeutic proteins including drugs which can either be dissolved or encapsulated within them forming a nanoparticle and a Nano capsule respectively. The delivered therapeutic proteins or drugs act by altering defective proteins or genes in the patient's cells. The size of the polymer nanoparticle could be tuned to enable these drugs and therapeutic protein to fit in. PNPs, like all nanoparticles are capable of regaining their size once inside the cell through the physiological change in $\mathrm{pH}$. They are also great candidates for gene delivery, because they are highly stable and they offer controlled release of active ingredients.

PNPs are synthesized from non-toxic biodegradable, biocompatible polymers like. These polymers can be used on their own to synthesize (PNPs) or they could be combined together to get better properties of nanoparticles. Polymer nanoparticles are used to overcome the various challenges that have been encountered in using gene therapy. Some genes have relatively long base sequences which make it difficult for them to be delivered to the desired sites. Moreover, the nucleases in the target cells may also degrade the DNA being delivered. And because the gene and the carrier are usually conjugated, their separation at the point of delivery is sometimes difficult.

Dendrimers for gene delivery, dendrimers are 1-10 nm, three dimensional globular synthetic macromolecules. They are highly branched and characterized by monodispersity. Terminal functional groups at the surface of the dendrimers, dictate the properties of the dendrimer macromolecule.

The properties of dendrimers, such as monodispersity, well-defined structure and the extensive quantity of surface functional groups, made them valuable tools to be used in gene delivery. Moreover, they can interact with DNA, RNA and antisense oligonucleotide through electrostatic interaction to form complexes that condense the nucleic acid. Hyper branched dendrimers are more suitable to be used as gene delivery tools than more structured dendrimers as their flexibility allows them to form more compact complexes with DNA.

The most well studied dendrimer is polyamidoamine (PAMAM), which is characterized by high solubility and reactivity due to the presence of empty internal cavities and numerous functional groups at its periphery. The six-generation PAMAM dendrimers are widely used dendrimers as vectors for gene delivery.

Liposomes for gene delivery, liposomes are small vesicle-like structures that are formed by self-assembly through lipids energetic
Volume 10 Issue I - 2019

\author{
Hajj Adel Anis \\ Cedars Jebel Ali International Hospital, UAE
}

Correspondence: Hajj Adel Anis, Medical Oncologist at Cedars Jebel Ali International Hospital, 9370 Rue Lajeunesse, Montreal, UAE, Tel 438992 55I6, Email ahajj@dr.com

Received: January 02, 2019 | Published: January 08, 2019

interactions. Liposomes possess properties such as reduced toxicity, safe preparation and reduced risk of immunological rejection, which enable its use for non-viral gene delivery.

Cationic lipids, are more commonly used for gene delivery whereas anionic liposomes uses are constricted to other therapeutic macromolecules, because the positive charge of the liposomes binds to the negatively charged nucleic acids much easier. The use of cationic liposomes for gene delivery is advantageous as it is biodegradable after administration in vivo, biocompatible and its surface can be diversely modified when using pegylated lipids. The most commonly used cationic liposomes utilized for the delivery of nucleic acids include DOTMA, DOTAP and DC-Chol.

Magnetic nanoparticles, paramagnetic nanoparticles have been used as drug carriers. Their accumulation is guided in target tissues using strong magnetic fields, and they were successfully used in cancer treatment. Similarly, the same technique has been applied to gene vectors; a high throughput magnetofecion was able to offer a new tool for gene therapy that overcomes the drawbacks of in vivo gene therapy. Magnetofecion has improved the efficacy of conventional transfection methods in vitro and in vivo. The term "magnetofection" refers to this method of magnet-assist gene delivery; the gene is joined to a magnetic particle or transporter, consisting of an iron-oxide encapsulated within a polymer or metallic shell. Alternatively, the particle can be dispersed in a polymer matrix. The shell or matrix may then be functionalized through attaching amines, biotin, streptavidin or antibodies in order to achieve maximum efficiency.

Gold nanoparticles, gold nanoparticles (AuNPs), are known to be appropriate gene delivery vehicles. The optical and physicochemical properties that allow for easy transfection into cells as well as their unique biocompatibility that make them non-toxic. Moreover, AuNPs can be easily modified and custom made for optimum delivery and specificity. Several issues must be taken into consideration for successful gene delivery, particularly effective cargo condensation, cellular uptake, DNA stability and prevention of degradation from nucleases as well as efficient delivery of DNA into the nucleus for expression. Several studies have shown lysine coated AuNPs produce more potent transfection vectors, that are able to condense DNA.

Quantum dots for labeling genetic material, quantum dots (QDs) are crystalline nanoparticles with electrical and mechanical properties. QDs are highly luminescent, colloidal semiconductor Nano crystals. 
QDs have unique size-dependant properties, which make them highly attractive for applications in catalysis, phosphors, photovoltaic, light emitting diodes (LEDs) and biological labeling. Made of semiconductor, quantum dots can be excited which makes them suitable not only in monitoring the genes; they are capable of overcoming the challenge of gene silencing. Semiconductor quantum dots (QDs) can be used to deliver genes such as RNA interference (RNAi) which is capable of silencing genes in the cell which either cause a disease or interfere with the activation of the delivered genes and synthesis of the therapeutic proteins. Additionally, QDs have been used as siRNA delivery vehicles to silence a target gene, and as fluorescent probes to analyze intracellular imaging in vivo. ${ }^{1-6}$

\section{Conclusion}

Nanoparticles are rapidly emerging as therapeutic delivery vectors defined by size-dependent properties. They offer several advantages over the traditional drug-delivery systems and medical diagnostics but also pose considerable challenges for systemic applications. Gene delivery is one of the important applications of nanotechnology. The Physicochemical properties such as size, shape, surface morphology have been found to have significant influence on the gene-delivery efficacy of nanoparticles. Furthermore, when administered in vitro and in vivo, the efficiency of nanoparticles depends on a wide variety of other parameters, that is, transfection conditions, time of exposure, cell type...
The US Food and Drug Administration (FDA) has approved only a limited number of gene therapy products for use in the United States.

\section{Acknowledgments}

None.

\section{Conflicts of interest}

Author declares that there is no conflicts of interest.

\section{References}

1. Bharali DJ, Mousa SA. Emerging nanomedicines for early cancer detection and improved treatment: current perspective and future promise. Pharmacol Ther. 2010;128(2):324-335.

2. Zhao G, Rodriguez BL. Molecular targeting of liposomal nanoparticlesto tumor microenvironment. Int J Nanomedicine. 2013;8:61-71.

3. Jabir NR, Tabrez S, Ashraf GM, et al. Nanotechnology-based approaches in anticancer research. Int J Nanomedicine. 2012;7:4391-408.

4. Peer D, Karp JM, Hong S, et al. Nanocarriers as an emerging platform for cancer therapy. Nat Nanotechnol. 2007;2(12):751-760.

5. Kumar Bishwajit S, Amin ML. Nanoemulsions: increasing possibilities in drug delivery. European Journal of Nanomedicine. 2013;5(2):97-110.

6. KT Nguyen. Targeted nanoparticles for cancer therapy: promises and challenges. Journal of Nanomedicine \& Nanotechnology. 2011;2(5):103e. 